\title{
Performance improvement of optical negative feedback laser by reducing feedback loop length
}

\author{
Shota Sato $^{1 \text { a) }}$, Genta Aizawa ${ }^{1}$, Nobuhide Yokota ${ }^{1}$, and Hiroshi Yasaka ${ }^{1 b)}$
}

Abstract The Optical path length of an input/output optical waveguide in a Si ring filter is reduced to shorten the feedback loop length of an optical negative feedback laser. Thanks to the increase in phase margin in the optical negative feedback system, the spectral linewidth of a single-mode semiconductor laser can be reduced to $99.8 \mathrm{kHz}$

Keywords: semiconductor laser, spectral linewidth, negative feedback, Si filter, integration

Classification: Integrated optoelectronics

\section{Introduction}

Compact and narrow spectral linewidth semiconductor lasers are required for digital coherent optical transmission systems [1]. Many studies have been conducted to develop such lasers $[2,3,4,5,6,7,8,9,10,11,12,13,14,15,16$, $17,18,19,20,21,22,23]$, and almost all of them have focused on increasing the laser cavity quality factor (Qfactor) by lengthening the laser cavities via the addition of external cavities. As a result, the cavity sizes of these lasers become long. Moreover, frequency noise increases at the repetition frequency coincident with round trip frequency of the external cavity modes, which makes it difficult to use the lasers in optical transmission systems. While a current feedback technique that does not rely on increasing the cavity Q-factor has been proposed [24, 25], it requires large feedback electrical circuits and the size of the laser system consequently becomes too large. To achieve a compact and very narrow spectral linewidth semiconductor laser, we have previously proposed an optical negative feedback semiconductor laser $[26,27,28,29]$ that features a simple structure consisting of a single mode semiconductor laser and an optical filter. The optical filter acts as an optical frequency discriminator and converts the frequency noise of the semiconductor laser into electrical field amplitude modulated feedback light that is then fed back to the singlemode semiconductor laser. In the optical negative feedback laser, the optical filter does not constitute the laser cavity, so the laser is free from the issue of frequency noise increase. By using a ring optical filter with a Si optical waveguide, a compact optical negative feedback laser with

\footnotetext{
${ }^{1}$ Research Institute of Electrical Communication, Tohoku University, Sendai 980-8577, Japan

a)s-shota@riec.tohoku.ac.jp

b) yasaka@riec.tohoku.ac.jp
}

DOI: 10.1587/elex.17.20190750

Received December 25, 2019

Accepted January 22, 2020

Publicized January 31, 2020

Copyedited February 25, 2020

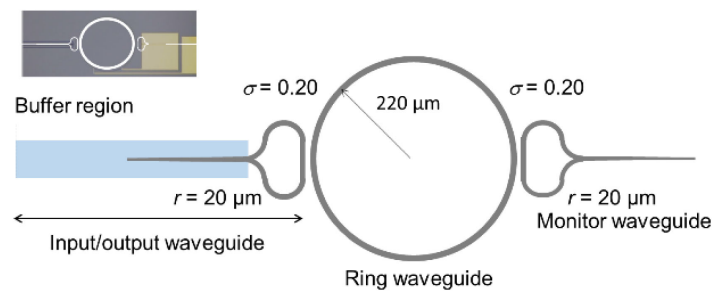

Fig. 1. Schematic structure of Si ring filter with reduced feedback loop length.

the spectral linewidth of $160 \mathrm{kHz}$ has been achieved [30]. The value of the reduced spectral linewidth, however, is limited by the small phase margin of the negative feedback system. In this paper, we report a new Si ring filter with a shorter input/output optical waveguide that helps reduce the feedback loop length and enlarge the phase margin of the negative feedback system. By applying the Si ring filter to a single-mode semiconductor laser, the spectral linewidth can be reduced to $99.8 \mathrm{kHz}$.

\section{Si ring filter and feedback characteristics}

Fig. 1 shows the schematic structure of the proposed ring filter with the Si optical waveguide. A single-mode semiconductor laser is coupled to the Si ring filter at left in the figure by mean of a butt coupling technique. In order to increase the coupling efficiency between the two, a $\mathrm{SiO}_{\mathrm{x}}$ slab buffer region and taper $\mathrm{Si}$ waveguide are located at the facet of the filter. The dimensions of the $\mathrm{Si}$ waveguide are set to $400 \mathrm{~nm}$ (width) and $220 \mathrm{~nm}$ (thickness) to satisfy the single-mode condition for 1550-nm transverse-electric polarized light. Propagation loss of the $\mathrm{Si}$ waveguide is assumed to be $3.6 \mathrm{~dB} / \mathrm{cm}$. Input light from the laser is coupled to the ring waveguide and re-coupled to the laser via input/output waveguide. The radius of the wrapping waveguide of the input/output waveguide $r$ is set to $20 \mu \mathrm{m}$ to avoid the increase in propagation loss at the curved waveguide. The total round trip waveguide length (feedback loop length) is reduced to $L_{\mathrm{FBL}} \sim 828 \mu \mathrm{m}$, which corresponds to the feedback loop delay time of $\tau_{\mathrm{FBL}} \sim$ $9.9 \mathrm{ps}$. The value can be reduced to almost half the value of the previous Si ring filter $\left(L_{\mathrm{FBL}} \sim 1226 \mu \mathrm{m}\right.$ and $\tau_{\mathrm{FBL}} \sim$ $18.8 \mathrm{ps}$ ) [30]. The radius of the ring waveguide is set to $220 \mu \mathrm{m}$ and the free spectral range of the filter is $50 \mathrm{GHz}$. The power transition efficiency of optical coupling between the input/output and ring waveguides $\sigma$ is set to 0.2 by setting the gap between the two waveguides to $420 \mathrm{~nm}$. The 


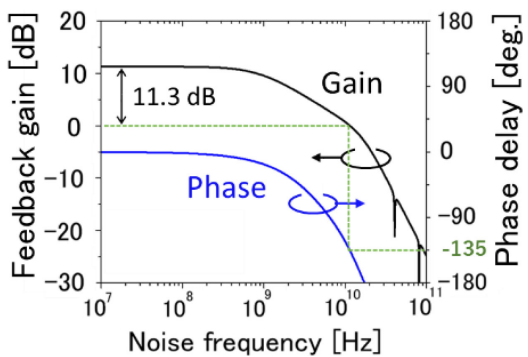

Fig. 2. Calculated feedback gain and phase of the optical negative feedback system of the laser as a function of noise frequency.

waveguide at the right of the ring waveguide is attached in order to monitor the condition of the butt joint between the single-mode semiconductor laser and the Si ring filter. The finesse of the Si ring filter is estimated to be 13.9, which is the same as the filter in the previous report [30]. The maximum conversion efficiency from frequency change to electrical field reflection coefficient of the filter is estimated to be $1.02 \times 10^{-10} \mathrm{~Hz}^{-1}$. We estimate the feedback gain and phase delay of the optical negative feedback system of the laser by calculating the transfer function of the system [27]. Fig. 2 shows the calculated result. We found that the feedback gain of the optical negative feedback system of the laser could reach $11.3 \mathrm{~dB}$ at the noise frequency range below several hundred mega hertz even when the phase margin of the system was set to 45 degrees. This indicates that the spectral linewidth of the optical negative feedback laser using the $\mathrm{Si}$ ring filter proposed here can reduce the value to two orders of magnitude narrower than the value under the free running condition. The laser's spectral linewidth can be reduced to less than $100 \mathrm{kHz}$ when a semiconductor laser with $10 \mathrm{MHz}$ spectral linewidth under a free running condition is used.

\section{Experiment}

We tested the optical negative feedback laser by integrating a distributed feedback (DFB) laser and the fabricated Si ring filter as a hybrid. Cavity length and threshold current $I_{\text {th }}$ of the DFB laser were $300 \mu \mathrm{m}$ and $9 \mathrm{~mA}$, respectively. The dimensions of the fabricated Si ring filter are the same as the one explained in the previous section. The characteristics of the filter are almost the same as the previous one [30] as predicted from the numerical analysis mentioned above. Fig. 3(a) shows a photograph of the integrated optical negative feedback laser. The gap between DFB laser and input/output waveguide of the Si ring filter is filled with refractive index matching gel to reduce the reflections from the facets of the two. Fig. 3(b) show the near field beam pattern of the output from the Si ring filter. It exhibits a clear single transverse mode pattern and indicates that the butt coupling between the DFB laser and the Si ring filter is done sufficiently. The spectral linewidth of the laser is measured using a delayed-heterodyne method [31]. A delay optical fiber of $20 \mathrm{~km}$ is used and the resolution of the measured system is estimated to be $5 \mathrm{kHz}$. Fig. 4(a) shows the measured beat spectra of the laser under free running and feedback conditions. Bias current to the DFB laser is set to $10 I_{\text {th }}$. The spectral line- (a)

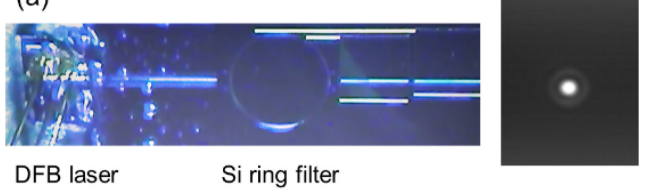

Fig. 3. (a) Photo of optical negative feedback laser consisting of DFB laser and Si ring filter. (b) Near field pattern of the output from Si ring filter's monitor waveguide.
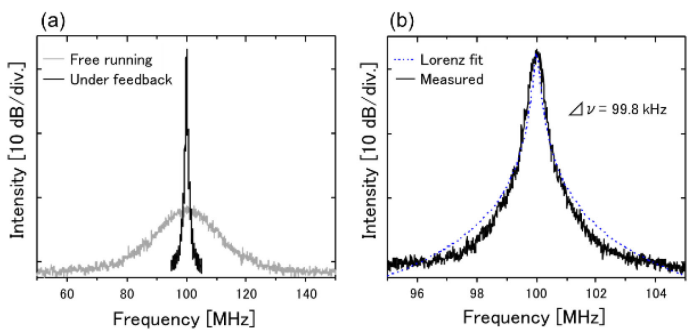

Fig. 4. (a) Measured beat spectra of lasing mode under free running (gray curve) and optical negative feedback (black curve) conditions. (b) Magnified beat spectrum of the optical negative feedback laser. Dotted curve is a fitted one by using Lorenz function.

width of the DFB laser is estimated to be around $10 \mathrm{MHz}$ under the free running condition. This can be reduced dramatically by performing optical negative feedback with the $\mathrm{Si}$ ring filter. Fig. 4(b) shows the magnified beat spectrum of the laser under the optical negative feedback condition. The dotted curve in the figure is a fitted one of the measured data using the Lorenz function. The reduced spectral linewidth is estimated to be reached to $99.8 \mathrm{kHz}$, which is two orders of magnitude narrower than the value under the free running condition $(\sim 10 \mathrm{MHz})$. The reduction ratio of the spectral linewidth is almost identical to the predicted value from the numerical calculation.

\section{Summary}

In this work, we improved the spectral linewidth reduction ratio in an optical negative feedback laser with a Si ring filter by reducing the optical path length of the input/ output waveguide of the $\mathrm{Si}$ ring filter. Thanks to the increase in phase margin of the optical negative feedback system, the spectral linewidth of the optical negative feedback laser was reduced to $99.8 \mathrm{kHz}$, which is two orders of magnitude narrower than the value for the free running DFB laser used in this experiment.

\section{Acknowledgment}

This work was supported by the Japan Society for the Promotion of Science through the Grants-in-Aid for Scientific Research under Grant 19H02185.

\section{References}

[1] M. Seimetz: "Laser linewidth limitations for optical systems with high-order modulation employing feed forward digital carrier phase estimation," OFC/NFOEC Dig. Tech. Papers (2008) OTuM2 (DOI: 10.1109/OFC.2008.4528637).

[2] O. Nilsson, et al:: "Oscillation frequency, linewidth reduction and 
frequency modulation characteristics for a diode laser with external grating feedback," Electron. Lett. 17 (1981) 589 (DOI: 10.1049/ el:19810415).

[3] K. Kikuchi, et al.: "Simple formula giving spectrum-narrowing ratio of semiconductor-laser output obtained by optical feedback," Electron. Lett. 18 (1982) 10 (DOI: 10.1049/el:19820008).

[4] K. Kojima, et al.: "Analysis of the spectral linewidth of distributed feedback laser diode,” J. Lightwave Technol. 3 (1985) 1048 (DOI: 10.1109/JLT.1985.1074295)

[5] H. Sato and J. Ohya: "Theory of spectral linewidth of external cavity semiconductor lasers," IEEE J. Quantum Electron. 22 (1986) 1060 (DOI: 10.1109/JQE.1986.1073086).

[6] B. Dahmani, et al:: "Frequency stabilization of semiconductor lasers by resonant optical feedback," Opt. Lett. 12 (1987) 876 (DOI: 10.1364/OL.12.000876).

[7] H. Li and H. R. Telle: "Efficient frequency noise reduction of GaAlAs semiconductor lasers by optical feedback from an external high-finesse resonator," IEEE J. Quantum Electron. 25 (1989) 257 (DOI: 10.1109/3.18538).

[8] P. H. Laurent, et al.: "Frequency noise analysis of optically selflocked diode lasers," IEEE J. Quantum Electron. 25 (1989) 113 (DOI: 10.1109/3.29238).

[9] M. F. Ferreira, et al:: "Noise and modulation performance of Fabry-Perot and DFB semiconductor lasers with arbitrary external optical feedback," IEE Proc. J Optoelectron. 137 (1990) 361 (DOI: 10.1049/ip-j.1990.0063).

[10] T. Kimoto, et al:: "Reduction of spectral-linewidth in high power SOA integrated wavelength selectable laser," IEEE J. Sel. Topics Quantum Electron. 11 (2005) 919 (DOI: 10.1109/JSTQE.2005. 853781).

[11] H. Ishii, et al.: "Spectral linewidth reduction in widely wavelength tunable DFB laser array," IEEE J. Sel. Topics Quantum Electron. 15 (2009) 514 (DOI: 10.1109/JSTQE.2008.2010237).

[12] H. Ishii, et al.: "Narrow spectral linewidth operation $(<160 \mathrm{kHz})$ in widely tunable distributed feedback laser array," Electron. Lett. 46 (2010) 714 (DOI: 10.1049/el.2010.0802)

[13] W. Liang, et al.: "Whispering-gallery-mode-resonator-based ultranarrow linewidth external-cavity semiconductor laser," Opt. Lett. 35 (2010) 2822 (DOI: 10.1364/OL.35.002822).

[14] K. Kasai, et al.: "A ${ }^{13} \mathrm{C}_{2} \mathrm{H}_{2}$ frequency-stabilized $\lambda / 4$-shifted DFB laser diode with an external fiber ring cavity having a linewidth of $2.6 \mathrm{kHz}$ and RIN of $-135 \mathrm{~dB} / \mathrm{Hz}$," CLEO Dig. Tech. Papers (2011) CTuV4 (ISSN: 2160-8989) (DOI: 10.1364/CLEO_SI.2011. CTuV4).

[15] W. Loh, et al.: "Packaged, high-power, narrow-linewidth slabcoupled optical waveguide external cavity laser (SCOWECL)," IEEE Photon. Technol. Lett. 23 (2011) 974 (DOI: 10.1109/LPT. 2011.2146245).

[16] Y. Zhao, et al:: "Hertz relative linewidth DFB laser with highfinesse cavity external optical feedback," CLEO Dig. Tech. Papers (2013) CTh1G.7 (ISSN: 2160-8989) (DOI: 10.1364/CLEO_SI.2013. CTh1G.7).

[17] K. Sato, et al.: "High output power and narrow linewidth silicon photonic hybrid ring-filter external cavity wavelength tunable lasers," ECOC Dig. Tech. Papers (2014) PD.2.3 (DOI: 10.1109/ ECOC.2014.6964263).

[18] H. Debregeas, et al.: " $2 \mathrm{kHz}$ linewidth C-band tunable laser by hybrid integration of reflective $\mathrm{SOA}$ and $\mathrm{SiO}_{2}$ PLC external cavity," ISLC Dig. Tech. Papers (2014) 50 (DOI: 10.1109/ISLC. 2014.158)

[19] R. Tang, et al:: "Narrow-spectral-linewidth silicon photonic wavelength-tunable laser with highly asymmetric Mach-Zehnder interferometer," Opt. Lett. 40 (2015) 1504 (DOI: 10.1364/OL.40. 001504).

[20] T. Komljenovic, et al.: "Widely tunable narrow-linewidth monolithically integrated external-cavity semiconductor lasers," IEEE J. Sel. Topics Quantum Electron. 21 (2015) 1501909 (DOI: 10.1109/ JSTQE.2015.2422752)

[21] T. Kita, et al:: "Narrow spectral linewidth silicon photonic wavelength tunable laser diode for digital coherent communication system,’ IEEE J. Sel. Topics Quantum Electron. 22 (2016) 1500612
(DOI: 10.1109/JSTQE.2016.2559418).

[22] H. Ishii, et al:: "Narrow linewidth operation $(<10 \mathrm{kHz})$ in selfinjection-locked tunable DFB laser array (SIL-TLA) integrated with optical feedback planar lightwave circuit (PLC)," ECOC Dig. Tech. Papers (2016) Tu.2.E.5

[23] F. Wei, et al.: "Subkilohertz linewidth reduction of a DFB diode laser using self-injection locking with a fiber Bragg grating FabryPerot cavity," Opt. Express 24 (2016) 17406 (DOI: 10.1364/OE.24. 017406).

[24] M. Ohtsu, et al.: "Linewidth reduction of a semiconductor laser by electrical feedback," IEEE J. Quantum Electron. 21 (1985) 1905 (DOI: 10.1109/JQE.1985.1072610).

[25] M. Ohtsu, et al:: "FM noise reduction and subkilohertz linewidth of an AlGaAs laser by negative electrical feedback," IEEE J. Quantum Electron. 26 (1990) 231 (DOI: 10.1109/3.44954).

[26] H. Yasaka, et al.: "FM noise and spectral linewidth reduction by incoherent optical negative feedback," IEEE J. Quantum Electron. 27 (1991) 193 (DOI: 10.1109/3.78220).

[27] K. Aoyama, et al.: "Optical negative feedback for linewidth reduction of semiconductor lasers," IEEE Photon. Technol. Lett. 27 (2015) 340 (DOI: 10.1109/LPT.2014.2371074).

[28] K. Aoyama, et al.: "3-kHz spectral linewidth laser assembly with coherent optical negative feedback," IEEE Photon. Technol. Lett. 30 (2018) 277 (DOI: 10.1109/LPT.2017.2783365).

[29] K. Aoyama, et al:: "Strategy of optical negative feedback for narrow Linewidth semiconductor lasers," Opt. Express 26 (2018) 21159 (DOI: 10.1364/OE.26.021159).

[30] K. Aoyama, et al:: "Compact narrow-linewidth optical negative feedback laser with Si optical filter," Appl. Phys. Express 11 (2018) 112703 (DOI: 10.7567/APEX.11.112703).

[31] T. Okoshi, et al.: "Novel method for high resolution measurement of laser output spectrum," Electron. Lett. 16 (1980) 630 (DOI: 10.1049/el:19800437). 